\title{
Un peu de bon sens du monde politique pour sortir de l'impasse?
}

\author{
Jean-Pierre Grillet
}

Dr med., Spécialiste en Dermatologie et Allergologie, membre FMH

Le malaise des coûts de la santé est pratiquement universel, et entraîne forcément une crise qui se révèle chez nous, au grand jour, à travers cet épisode de révision du Tarif médical. Celle-ci a été pratiquement imposée par la Commission Fédérale des Finances il y a plus de 5 ans. Ce problème de tarif est naturellement intimement lié à celui des coûts de la santé, qui ont fortement augmenté pour de multiples raisons connues, et parfois moins évidentes. Comment en est-on arrivé à l'impasse actuelle? D'un côté, les médecins ont vu leur pouvoir d'achat diminuer fortement depuis des décennies, en bonne partie par une absence d'indexation des charges des cabinets médicaux, et de l'autre, les primes d'assurance-maladie ont augmenté de telle sorte qu'elles ont absorbé complètement les accroissements de salaires obtenus par les salariés du pays.

\section{Pas de prestations de première classe pour le prix du low cost}

En définitive, il s'agit d'un problème de démocratie: d'un côté les médecins (= la minorité) souhaitent une adaptation des prix de leurs prestations, parfaitement justifiée à de nombreux égards, alors que la population générale (= la majorité) ne supporte plus les augmentations et souhaite payer moins de primes d'assurance-maladie, donc payer moins pour la santé. Cette même population, soit dit en passant, n'a cependant pas dit qu'elle souhaitait des soins de moindre qualité en même temps qu'une baisse des prix... Il serait donc temps d'expliquer enfin qu'en matière de santé, comme dans les autres domaines, on ne peut pas avoir des prestations de première classe pour le prix du low cost. Ce que le Conseiller fédéral (CF) Berset ne peut pas ignorer, c'est le lien très étroit qui existe entre rémunération et qualité. Si certains de nos pays voisins ont des coûts de consultations inférieurs, il faut aussi voir le niveau de qualité des soins. Il ne sert à rien que Monsieur Berset mette sur pied des centres de contrôle de qualité s'il ne donne pas aux médecins les possibilités de réaliser cette qualité. Que les décideurs expriment enfin clairement s'ils mettent la prio- rité sur la qualité ou sur le prix! D’un autre côté, les brimades dont les médecins ont été la cible, nettement plus que d'autres acteurs de la santé, créent des situations de lassitude et de "ras-le-bol», avec perte de motivation, peu favorables aux conditions de développement de la qualité des soins attendue.

Le CF Alain Berset n'est pas stupide et il a très bien compris les enjeux. Sans doute ne serait-il pas contre une augmentation des revenus des médecins, si la situation n'était pas si tendue. Seulement voilà, il est un élu, tout comme les parlementaires, et, tout le monde le sait, il vaut mieux faire plaisir à la majorité des électeurs, si l'on souhaite être réélu. Il vaut donc mieux se fâcher avec la minorité représentée par les médecins qu'avec la majorité de la population et du Parlement.

\section{Pas de médecine sans médecin}

Seulement voilà, ce n'est pas si simple, et c'est même beaucoup plus compliqué. On ne pourra pas lister ici l'ensemble des éléments de ce dossier extrêmement touffu. Mais d'abord un fait est incontournable: il n'y a pas de chirurgie sans chirurgien, et il n'y a pas de médecine sans médecin. Et si le corps médical savait être un tout petit peu plus habile, il saurait faire mieux jouer cet argument en sa faveur. Bien sûr que certaines

\section{Il vaut donc mieux se fâcher avec la minorité} représentée par les médecins qu'avec la majorité de la population et du Parlement.

professions disparaissent (comme les chapeliers...) et d'autres ont vu leurs rémunérations considérablement diminuer. Mais il y a peu de professions qui se soient autant complexifiées et modifiées que la médecine et pour lesquelles il n'y a eu aucune adaptation de revenu depuis 2004. Pour ceux qui sont installés en pratique privée depuis trente ans, il n'y a jamais eu ni indexation ni revalorisation, alors que les charges ont largement augmenté, ce qui, en d'autres termes, signifie que leur pouvoir d'achat a baissé de pratiquement 30\% en 30 ans, alors que les revenus des salariés ont augmenté 
d'environ 10\% dans les 10 dernières années, selon l'Office Fédéral de la Statistique.

Si les médecins sont bien conscients que la situation économique n'est guère favorable à une augmentation des charges d'assurance pour les familles, ils sont aussi fatigués de devoir assumer les conséquences des décisions aberrantes du Parlement. Les coûts de fonctionnement des cabinets médicaux en personnel et en infrastructure ont été largement augmentés par des mesures imposées par Berne et qui n'étaient pas indispensables. Le Parlement s'est aussi illustré par des décisions incompréhensibles et des errements dans le dossier du moratoire sur l'ouverture des cabinets médicaux. L'interruption du moratoire (qui avait pour but inavoué de créer les conditions favorables à la fin de l'obligation de contracter, sous le poids du lobby des assureurs) n'a pas permis de résoudre le problème de manque de médecins dans les vallées; elle a, par contre, été la cause de l'explosion des coûts, du moins pour les cantons-ville et les cantons frontaliers, liée à des installations dont l'utilité n'était pas prouvée. C'est aussi le Parlement qui refuse depuis des années de mettre de l'ordre dans les comptes, toujours aussi opaques, des assurances-maladie, dont les primes ont grimpé bien plus que les coûts réels des soins. C'est donc maintenant au Parlement avant tout d'assumer ses erreurs et d'accorder une augmentation, et non pas de chercher de nouvelles punitions pour les médecins. Car si le CF Berset et le Parlement peuvent se permettre de contraindre notre corporation à des économies drastiques, en diminuant encore les revenus basés sur les indices de 1994, pourquoi n'en font-ils pas de même avec l'Administration fédérale? Les impôts pèsent aussi lourdement sur la population et le train de vie de nos administrations n'est pas toujours en adéquation avec les résultats fournis. En ramenant le nombre de fonctionnaires et les traitements de ceux-ci au niveau de ceux de 1994 (comme c'est le cas pour les médecins!), la Confédération pourrait réaliser des économies de quelques milliards qui pourraient résoudre le problème de la Santé dans notre pays.

\section{Le besoin de bon sens}

En définitive, il ne sert à rien que la fmCh se désolidarise de la FMH, et que MFE suisse refuse tout compromis, que la FMH fasse le grand écart pour essayer d'améliorer la situation de certains médecins tout en respectant les exigences du CF Berset, car cela revient de facto à baisser une ou plusieurs autres spécialités. Nous voyons bien dans quelle impasse la tentative de révision et la votation générale nous ont conduits et personne ne peut honnêtement prétendre que l'on va trouver une solution s'il n'y a pas de geste de bonne volonté et un peu de bon sens de part et d'autre. Cela

Il n'y a pas de chirurgie sans chirurgien, et il n'y a pas de médecine sans médecin.

signifie que l'on ne s'en sortira pas sans une négociation directe et bien menée avec le CF Berset, et un compromis sur une augmentation.

Comment donc continuer? De manière pragmatique, en nous montrant unis, en donnant un mandat clair et en soutenant de toutes nos forces notre président de la FMH, le Dr Jürg Schlup, qui a déjà démontré à plusieurs reprises ses compétences dans des négociations menées dans la discrétion mais néanmoins efficaces. Lui donner mandat pour tenter d'obtenir de notre ministre une compensation, au moins partielle, de l'augmentation de nos charges contre un remaniement de quelques positions, ce qui était, au fond, le but premier de la révision du TARMED. Celle-ci s'en est éloignée par l'adoption de positions de plus en plus rigides qui n'ont plus laissé le moindre espace au bon sens. Il est évident que sans celui-ci, toute solution est impossible et une épreuve de force, dont personne ne sortira vainqueur, est à craindre. 